\title{
IMPLANTAÇÃO DA METODOLOGIA DOS OKRS COMO FERRAMENTA DE MELHORIA ESTRATÉGICA. ESTUDO DE CASO: GRUPO DE CLÍNICAS ODONTOLÓGICAS
}

\author{
Luccas de Moraes Magalhães (UVA) luccas_magalhães@hotmail.com \\ Izabel Saldanha (UVA) izabel.saldanha@uva.br \\ Antônio Sarquis (UVA) sarquis@uva.br \\ Rafael Cunha (UCAM) rafael.a.cunha@ hotmail.com \\ Daiane Santos (UVA) daiane.santos@uva.br
}

\section{Resumo}

O mercado brasileiro vem sofrendo mudanças em sua estrutura se tornando cada vez mais competitivo, parte pela conjuntura econômica, pelo movimento econômico após passar por uma crise, parte pelas novas tecnologias, metodologias e inovações que mudam a forma de trabalhar. A estrutura apresentada faz com que as empresas de micro e pequeno porte tenham dificuldades para se adaptarem ao novo cenário corporativo e comecem a se preocupar com os setores de produto, tecnologia, vendas, financeiro e estratégico para sobreviver nos novos desafios. O trabalho em questão tem como foco a parte estratégica da empresa tendo como objetivo principal realizar um estudo de caso em um grupo de clínicas odontológicas demonstrando as melhorias geradas por uma gestão por desempenho com foco na metodologia OKR (Objective and Key Results) como método gerencial para desenvolvimento operacional e organizacional.

Palavras-Chaves: gestão do desempenho organizacional, Objectives and Key Results, clínicas odontológicas, indicadores de desempenho.

\section{Introdução}

O mercado corporativo brasileiro tem grande parte de sua composição de micro e pequenas empresas, sendo 27\% do PIB do Brasil (SEBRAE, 2017), além deste segmento de empresas ter uma grande taxa de mortalidade de $24,4 \%$ para empresas até 2 anos de nascimento como análise em 2016 do Sebrae. (SEBRAE, 2016) As empresas de micro e pequeno porte tem como o principal fator de mortalidade a parte estratégica de planejamento. (SEBRAE, 2016)

Segundo Aguiar (2002) deve-se conhecer profundamente o problema, e, ao mesmo tempo, empenhar-se em desdobrá-lo em problemas prioritários mais simples (AGUIAR, S. , 2002).Dentro do universo das pequenas empresas, uma marca que tem dois anos no mercado e está ligada ao serviço de clínicas ortodônticas que é sediada no Rio de Janeiro e ocupa a baixada carioca foi selecionada como empresa a ser analisada. Algumas de suas 
características que auxiliaram em sua escolha foram serviço básico para o povo, além de ter como foco a classe popular e o seu tempo de vida, que está no nicho apresentado anteriormente de empresas que necessitam de direcionamento e tem risco de fechamento nos dois primeiros anos. Assim a marca necessitava de indicadores chaves e um direcionamento para os resultados que se esperava alcançar. Como (GATES, 1999) pontua, a medição do desempenho foi definida como o procedimento de implementar a estratégia por meio da sua tradução em resultados entregáveis.

Para solucionar o problema de direcionamento, controle da parte estratégica e auxiliar o grupo de clínicas em seu desenvolvimento e passagem pelas adaptações do mercado após a recessão de 2016, o OKR (Objective and Key Results) foi selecionado como ferramenta de gestão. Segundo Larry Page CEO da Alphabet e fundador do Google, os OKRs dão muita visibilidade a uma organização. Eles também oferecem uma maneira produtiva de analisar de forma mais ampla o processo. A metodologia OKR estruturou os planejamentos de Larry Page na Google viabilizando a missão de organizar as informações do mundo, além de manter a empresa de Page no caminho certo quando mais importava. (DOERR, 2019)

Com isso, o estudo em questão apresentará o framework do OKR (Objective and key results) que é uma metodologia ágil estratégica e como uma gestão focada em performance auxiliou o grupo de clínicas odontológicas de pequeno porte a seu crescimento.

\section{Definição e aplicação do $O K R$}

O OKR, definido por Lamorte, é um framework de pensamento crítico e disciplina contínua que visa garantir que os funcionários trabalhem juntos, concentrando seus esforços para fazer contribuições mensuráveis que impulsionam a empresa para frente. (NIVEN, P. R., LAMORTE, B., 2016). Felipe Castro também define OKR (Objectives and Key Results) como um sistema para programação de objetivos usado pela Google e outras companhias. Ele é um simples método para criar alinhamento e engajamento para atingimento de objetivos ambiciosos e mensuráveis. (CASTRO, 2017)

A sigla OKR é um acrônimo para objetivos e resultados chave (Objective and Key Results). O objetivo é qualitativo, os resultados chaves (normalmente três) são quantitativos. Eles são utilizados para gerar foco em um grupo ou indivíduo para uma meta. O objetivo estabelece uma meta para um período específico de tempo, normalmente um trimestre. Os indicadores chave medem o quanto do objetivo foi alcançado no final do período. (WODTKE, C., 2016) 
Figura 1 - Ciclo OKR

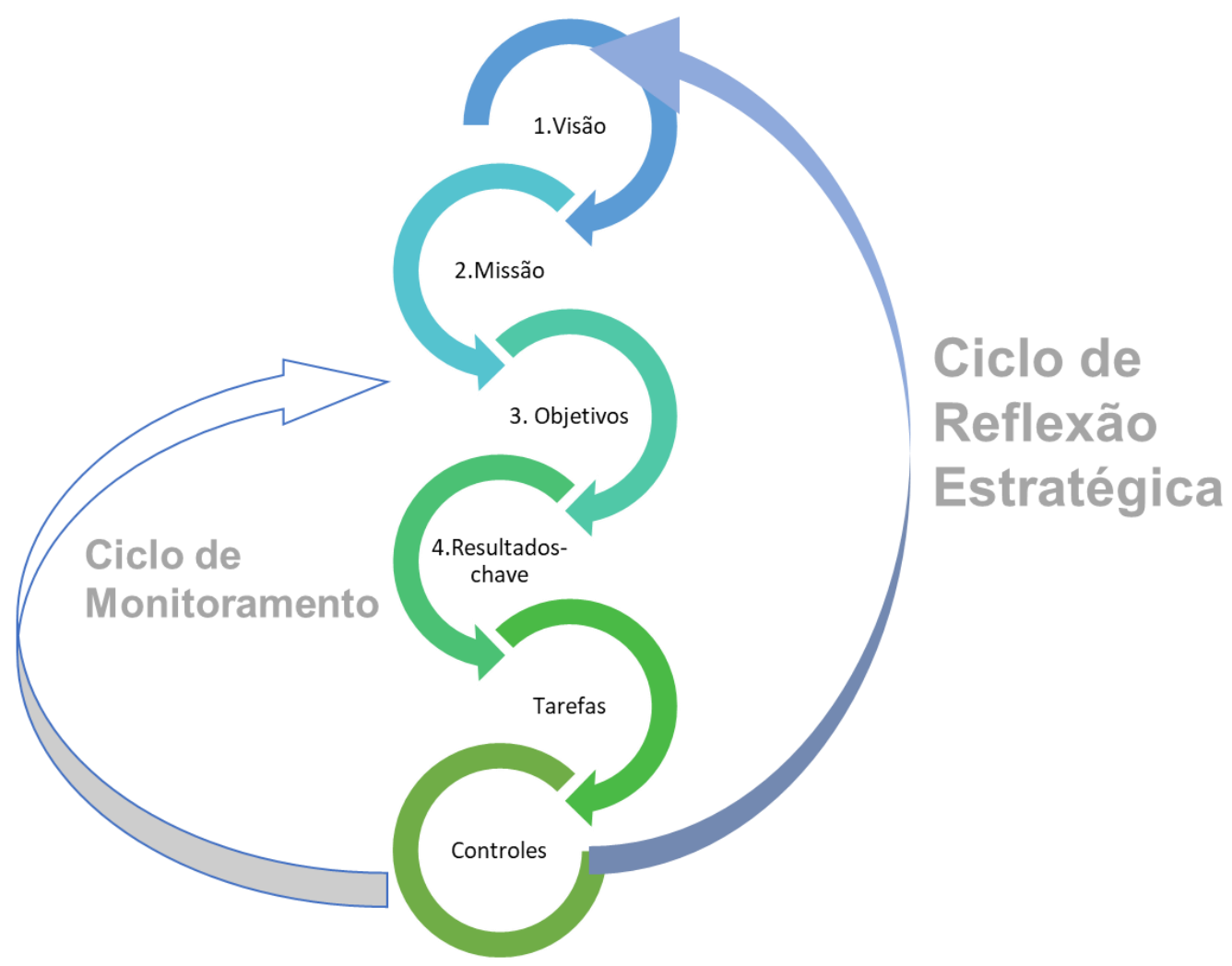

Fonte: (BETTERWORKS, 2015)

Logo, o OKR é uma ferramenta de análise crítica estratégica que alinha visão, objetivos da empresa com metas quantitativas, gerando indicadores chave para medir o desempenho e levar para os funcionários as questões como "aonde se quer chegar" e "como chegar no objetivo".

A BetterWorks pontua alguns dos benefícios que o OKR proporciona para a empresa que o utiliza, estes estão descritos na tabela 1 .

Tabela 1: 


\begin{tabular}{l}
\hline \multicolumn{1}{c}{ Benefícios que o OKR } \\
\hline Direcionar o pensamento disciplinado para que os objetivos principais sejam alcançados. \\
\hline Informar a todos o que é importante \\
\hline Gerar uma comunicação mais efetiva \\
\hline Estabelecer indicadores para mensurar progresso \\
\hline Concentrar os esforços e garantir alinhamento \\
\hline
\end{tabular}

Fonte: Elaborado pelos autores com base em BETTERWORKS, 2015

Em resumo o OKR pode ser definido na seguinte estrutura:

Eu vou [objetivo] medido por [Resultados-chave].

O objetivo deve ser significante para a companhia e pessoalmente "inspiracional". Ele deve ser alinhado e suportado por toda a organização. (BETTERWORKS, 2015).

Os objetivos respondem à pergunta de "aonde queremos chegar?" e "o que queremos fazer?" Niven define um objetivo como uma frase concisa que ambiciona uma meta ampla e qualitativa projetada para impulsionar a organização em uma direção desejada. (NIVEN, P. R., LAMORTE, B., 2016)

No OKR os objetivos têm como base a metodologia S.M.A.R.T ou M.A.R.T.E em português, que explica que para um objetivo ser bem estruturado deve ser mensurável, atingível, relevante, temporal e ser específico. (NIVEN, P. R., LAMORTE, B., 2016)

Abaixo estão três exemplos de objetivos da gerência de um time de futebol americano que John Doerr utiliza no OKR: (DOERR, 2019)

- $\quad$ Gerente geral de Futebol: fazer dinheiro para os donos.

- Técnico: Ganhar o campeonato

- $\quad$ Chefe das Relações Públicas: Ocupar 88\% das cadeiras do estádio.

Após escolhido os objetivos, se define os KR's ou indicadores chave que respondem o caminho que será seguido para atingir os objetivos. Eles são metas quantitativas que são 
acompanhadas periodicamente para ver o progresso dos objetivos. Os KR's são divididos em três tipos: 1) métricas de base; 2) métricas-alvo; e 3) marcos importantes (LAMORTE B., 2015)

O primeiro tipo de indicador tem como objetivo trazer o valor inicial para uma métrica de um novo processo que não tem histórico. O segundo tipo de indicador tem como objetivo atingir um valor destino. Lamorte ainda subdivide em três categorias:

- Positiva: Quanto maior melhor

- $\quad$ Negativa: Quanto menor melhor;

Limite: bandas máxima e mínima sem valor de atingimento;

(LAMORTE B., 2015)

Por fim, os marcos importantes são mais tarefas do que metas. Eles normalmente são divididos em métricas menores para sua medição. (LAMORTE B., 2015)

Os resultados chave são medidos variando de 0 até 1 , tendo como objetivo atingir 0,7 a 0,8 no trimestre, visto que são organizados para serem mais difíceis de atingir que uma meta normal. (LAMORTE B., 2015)

Teresa e Daniel ao realizarem o estudo do OKR na NTI-UFPE, discorreram que os objetivos do OKR precisam ser difíceis de serem alcançados de forma que $70 \%$ de seu atingimento já signifique que o objetivo está praticamente atingido, já agregou valor e está próximo de sua conclusão. (TERESA M M MACIEL, DANIEL ARCOVERDE, 2017)

O OKR é uma metodologia que tem sua aplicação e medição variáveis a partir da necessidade das organizações. Um exemplo, o Spotify usa um ciclo estratégico de 6 meses para seus objetivos, enquanto seus times utilizam medições a cada 6 semanas. Esta variabilidade gera histórias e visões interessantes depois de cada tentativa de organização individual. (CASTRO, 2017)

A maioria das empresas e times tentam utilizar OKRs mensais como uma lista a ser completada. Quando equipes querem mensurar valor, a frequência trimestral se torna a mais 
lógica, visto é necessário tempo para desenvolver as iniciativas e mensural o real impacto delas. (CASTRO, 2017)

Como uma regra geral, quanto menor a cadência do OKR, menor o limite de sobrecarga a ser atingido. Quanto mais longa for a cadência, menores serão as incertezas do negócio. (CASTRO, 2017)

A Figura 2, a seguir, apresenta o exemplo de John Doerr sobre o time de futebol:

Figura 2 - OKR no time de futebol americano

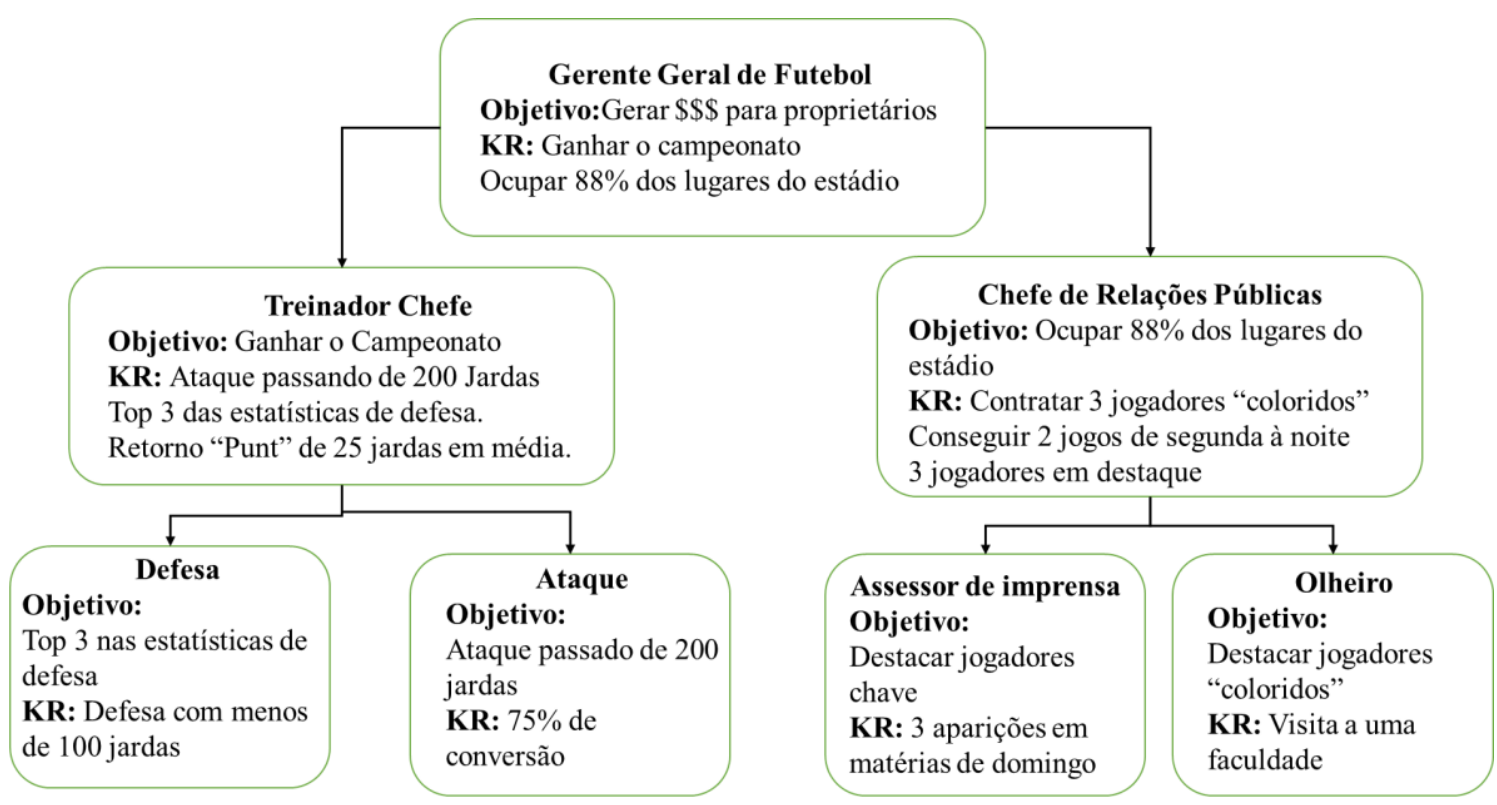

Fonte: (DOERR, 2019, p. 84)

Como se pode ser verificado na figura 2, o OKR trabalha com o objetivo vindo da área estratégica (Gerente Geral de futebol), define seus resultados chave para atingir o objetivo e os resultados chave se tornando os objetivos dos associados diretamente ligados ao Gerente. Logo, o OKR começa com a missão e visão da empresa, conectando os objetivos da alta gerência e simplificando-o em metas que serão se tornarão o objetivo da área inferior, até toda a hierarquia ter suas métricas definidas e alinhadas a visão e missão da empresa.

Para Lamorte os OKRs para serem efetivos devem ter dez características, como pode ser visto na tabela 2 
Tabela 2: Características das $O K R s$

\begin{tabular}{|c|c|c|}
\hline Características das OKRs & $\begin{array}{l}1 . \\
2 . \\
3 . \\
4 . \\
5 . \\
6 . \\
7 . \\
8 . \\
9 . \\
10 .\end{array}$ & $\begin{array}{l}\text { Resultado não tarefas; } \\
\text { SMART/MARTE; } \\
\text { Focado em poucos objetivos; } \\
\text { Espírito de Dono do criador do OKR; } \\
\text { Alinhamento para sucesso; } \\
\text { Continuamente atualizado; } \\
\text { Linguagem Clara; } \\
\text { Quanto maior melhor, ser positivo; } \\
\text { Ter clara a segmentação do dono e } \\
\text { Inspiracional. }\end{array}$ \\
\hline
\end{tabular}

Fonte: Elaborado pelos autores com base em LAMORTE B.,, 2015

Por fim, Dick Costolo, CEO do Twitter, foi perguntado em uma entrevista sobre o que aprendeu no Google e que aplicou no Twitter. Ele respondeu que os OKRs foram o que foi aprendido no Google que ele definitivamente aplicou no Twitter. Os OKRs são um grande caminho que ajuda todos da companhia a entender o que é importante e como mensurar o que importa. O OKR é uma grande forma de comunicar estratégia e como mensurar o seu impacto nela. Ele ainda concluiu que quando se está crescendo a comunicação é a estrutura mais difícil de escalar. Logo o OKR é a forma de todos entenderem como mensurar sucesso e estratégia (LAMORTE B., 2015).

O OKR como proposto na introdução deste estudo, pode ser aplicado tanto em grandes estruturas como em pequenas estruturas como Startups, por seu uma metodologia estruturalmente mais simples.

\section{Metodologia}

Segundo Mascarenhas (2012) a metodologia serve para explicar o que foi realizado durante o estudo e como foi realizado, tendo como objetivo descrever o método, participantes, o tipo de 
pesquisa e os instrumentos utilizados na pesquisa. A metodologia pode ser classificada pelas bases que a estruturam, como as bases lógicas da investigação, a abordagem do problema, o objetivo geral, o propósito da pesquisa e o procedimento técnico. (MASCARENHAS, 2012)

Este estudo foi desenvolvido através de um levantamento bibliográfico sobre a metodologia $O K R$, para fundamentar escolhas de sistemas de gestão mais adequados para a estrutura do mercado de clínicas ortodônticas existente. Pela abordagem do problema, a pesquisa qualitativa com embasamento quantitativo foi utilizada para maior profundidade com o problema (qualitativo) ao mesmo tempo que a coleta de dados, análise e tratamento geraram maior confiabilidade e capacidade de generalização (quantitativo).

$\mathrm{Na}$ pesquisa foram utilizadas premissas vindas das informações, dados coletados para montagem de planos de ação e conclusões mais abrangentes como serão apresentadas nos seções 4 e 5, fazendo o método indutivo a melhor base lógica de investigação (MASCARENHAS, 2012).Sendo assim, a pesquisa realizada será um estudo de caso com pesquisa bibliográfica, usando o método indutivo, utilizando abordagens qualitativas e quantitativas, avaliando resultados, diagnosticando as clínicas e propondo planos de ação.

\section{Implementação da metodologia}

De acordo com pesquisa com os diretores da empresa foram identificados gargalos como controle das clínicas, métricas de direcionamento e comunicação. Para decidir o melhor framework a ser implementado, se observou as possíveis causas raízes dos problemas mencionados. No caso do controle das clínicas foi implementado uma maior frequência de controle das receitas passando de mensal para diária, além da segmentação de cada receita por sua fonte originária. Nas métricas de direcionamento foi detectado uma necessidade de comunicação sobre o que se esperava das clínicas e aonde se queria chegar, logo a implementação de métodos e ferramentas para se alcançar o resultado desejado e a definição de objetivos para as clínicas eram indispensáveis. Por fim, a comunicação da empresa por ter as adversidades encontradas nas métricas de direcionamento e nos controles estava fragmentada, sendo crítico ter ações para gerar alinhamento e confiança em todas as pontas do processo.

Assim, o OKR um framework de gestão como mencionado no subcapítulo 2 foi escolhido para ser o alicerce do estudo, visto que direciona o pensamento disciplinado para que os objetivos principais sejam alcançados, informa a todos o que é importante, gera uma 
comunicação mais efetiva, como também garante maior alinhamento e estabelecimento de indicadores para mensurar o processo.

Para iniciar a implementação do OKR é fundamental desdobrar a visão, missão e valores da empresa em objetivos a serem alcançados na clínica. Porém, os objetivos e metas necessitam ser específicos e bem detalhados para o modelo. Portanto, a primeira implementação do estudo foi a criação das planilhas online que seriam compartilhadas via google drive onde se transcreveria o realizado no dia em valores e quantidade conforme a figura 8, consolidando as informações diariamente e as compilando no final do mês para análise em conjunto com as informações do sistema.

Figura 3 - Primeira planilha online

\begin{tabular}{|l|}
\hline Conta \\
\hline Bruto Dia \\
\hline R\$ Cartão Crédito dia \\
\hline R\$ Cartão Débito dia \\
\hline R\$ Dinheiro dia \\
\hline Despesas \\
\hline Cancelamentos dia \\
\hline Avaliações no dia \\
\hline Demais Mensalidades \\
\hline Vendas \\
\hline Total de contratos fechados desde inauguração \\
\hline Total de pacientes ativos \\
\hline Total de pacientes inativos \\
\hline Quantidade de agendados no dia \\
\hline Clientes confirmados no dia \\
\hline Clientes que compareceram dia \\
\hline Atendimentos dia \\
\hline Primeiras Mensalidades \\
\hline Segundas Mensalidades \\
\hline Raspagens no dia \\
\hline Pagamentos de meses anteriores \\
\hline Cofre \\
\hline Depósitos \\
\hline Novos clientes dia \\
\hline Clientes SEM contato \\
\hline
\end{tabular}


Nos meses de setembro e outubro de 2018, somente foi controlado o preenchimento e repasse de ajustes para geração de histórico de informações sobre planos vendidos, segundas mensalidades e demais mensalidades.

No início de novembro foram definidos os objetivos estratégicos de forma qualitativa e o desdobramento de metas quantitativas no espaço de dois meses para o fechamento do ano de 2018 .

Figura 4 - OKR 2018

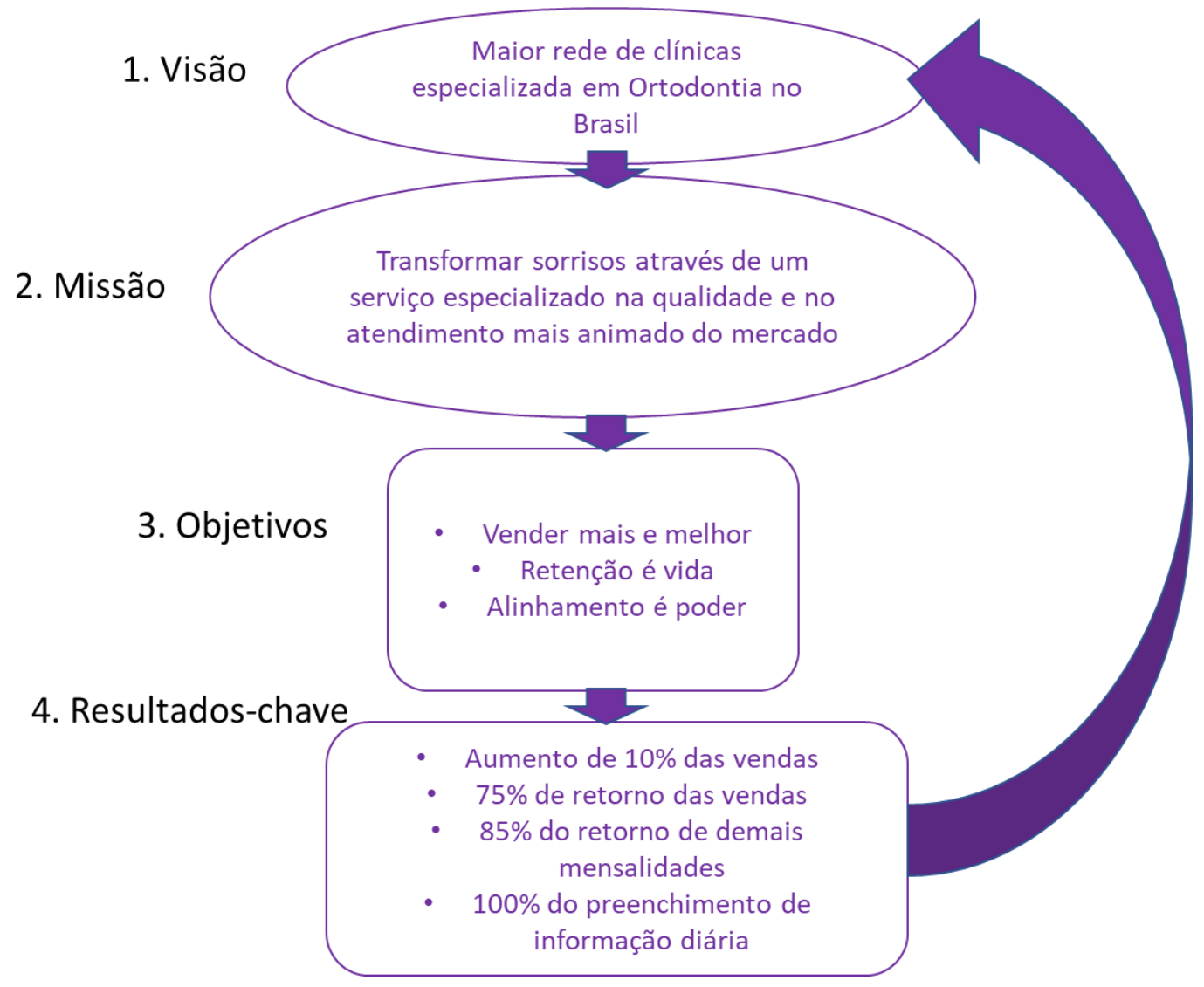

Fonte: Elaboração Própria (2019)

Na figura 4, a partir da visão e missão foram desdobrados três objetivos sendo eles: vender mais e melhor, retenção é vida e alinhamento é poder. Os objetivos foram quantificados em 4 indicadores de desempenho diretamente ligados com a visão da empresa.

Para a definição inicial dos indicadores foi utilizado os dados históricos de setembro e outubro, como o modelo de retenção e visão de crescimento dos diretores da empresa. Assim, 
a carteira de clientes mais antigo deveria ter no máximo $15 \%$ de perda e $25 \%$ no primeiro retorno a unidade, além de um crescimento de $10 \%$ das vendas para um aumento do bruto da clínica.

Para a comunicação dos objetivos e novas métricas, foi realizada uma reunião mensal que juntava todos os gerentes das clínicas. Além da passagem das novas métricas, foi definido um processo de acompanhamento semanal que consistia em uma reunião por telefone do atingimento dos indicadores e definição de planos de ação para melhoria do direcionamento, acarretando um maior alinhamento de expectativa e realidade.

\subsection{Controle e Revisão dos OKRs}

Em janeiro de 2019, a partir dos dados gerados no final de 2018 foi projetado o faturamento de dezembro de 2019. Com a meta anual definida se segmentou o crescimento mensal para cada clínica e criou-se o controle de Real x meta. Para atingir o objetivo de receita para 2019, foram revistos todos os objetivos e metas das clínicas e definido o prazo de 6 a 8 meses para retorno do estudo. Com isso, os OKRs de 2019 foram definidos e são representados na imagem abaixo:

Figura 5 - OKR 2019.1

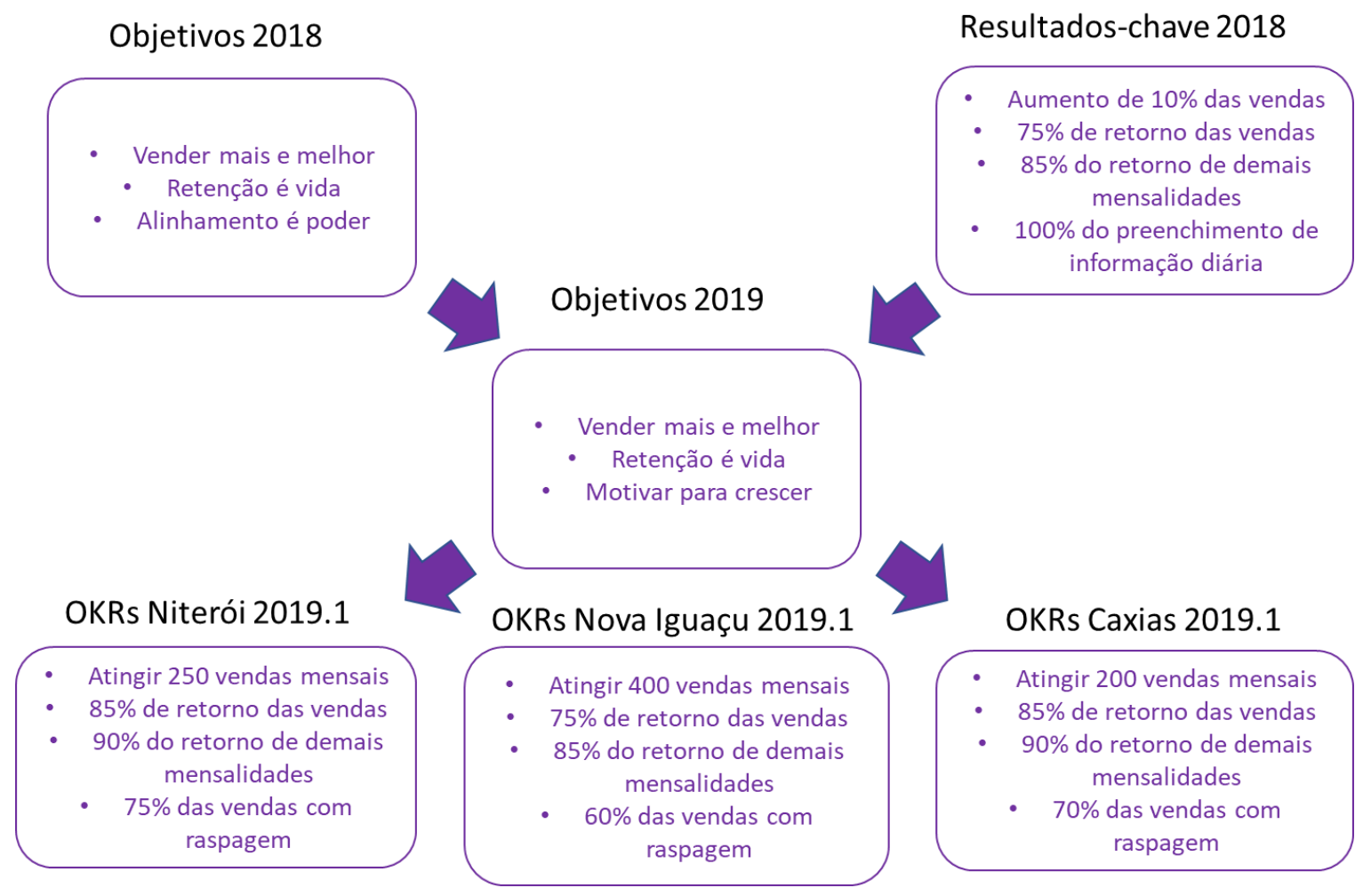


Como ilustrado na Figura 5, os objetivos de 2019 foram modificados em reunião estratégica com os diretores da empresa. Foi definido que o modelo de alinhamento já estava sendo executado, logo motivar os colaboradores a continuar o crescimento da clínica seria o mais indicado. Para motivar a continuidade de desenvolvimento foram revistas as comissões de vendas, bonificações para o time administrativo e benefícios trimestrais pelo melhor desempenho, além da criação de um plano de carreira gerando maior engajamento para o atingimento das metas. Os objetivos mantidos de 2018 foram intensificados nos resultadoschave, outra revisão de indicadores proposto pelo modelo OKR que tem como grande vantagem o dinamismo.

As metas propostas foram estruturadas a partir do modelo S.M.A.R.T no qual elas deveriam ser alcançáveis, mensuráveis, específicas, relevantes e temporais. A tabela 3 que segue abaixo especifica os resultados de 2018 em quantidade das segmentações da receita.

Tabela 3 - Quantidade de Receita

\begin{tabular}{l|c|c|c|c|c|c|c|c}
\hline NITEROI & Set/2018 & \% & Out/2018 & \% & Nov/2018 & \% & Dez/2018 & \% \\
\hline VENDAS & 223 & & 228 & & 198 & & 247 & \\
\hline $1^{\mathbf{o}}$ RETORNO & 164 & & 136 & $61 \%$ & 184 & $81 \%$ & 174 & $88 \%$ \\
\hline DEMAIS RETORNOS & 516 & & 487 & $72 \%$ & 601 & $96 \%$ & 703 & $90 \%$ \\
\hline RASPAGENS & 55 & & 92 & & 105 & & 177 & \\
\hline
\end{tabular}

Fonte: Elaboração Própria (2019)

Com os objetivos para 2019 e os dados de 2018, além do referencial teórico do OKR e modelo de negócios de retenção se definiu como difícil atingir mensalmente no primeiro semestre de 2019 os valores apresentados na figura 5 para cada clínica do estudo. Para melhorar a clareza da meta, controle e comunicação por parte do time operacional os controles de 2018 foram aperfeiçoados como ilustrado na figura 6.

Figura 6 - Controle 2019.1

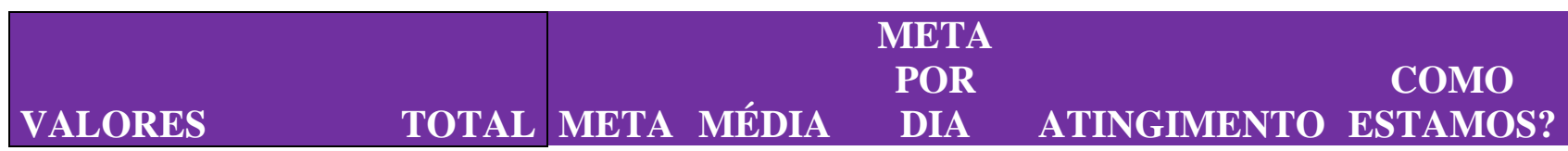




\begin{tabular}{|c|c|c|c|c|c|c|}
\hline VENDAS & & & & & & \\
\hline MANUNTENÇÕEs & & & & & & \\
\hline RASPAGENS & & & & & & \\
\hline INST.INFERIOR & & & & & & \\
\hline HIRAX & & & & & & \\
\hline KITS & & & & & & \\
\hline $\begin{array}{l}\text { CLAREAMENTO } \\
\text { CASEIRO }\end{array}$ & & & & & & \\
\hline $\begin{array}{l}\text { CLAREAMENTO } \\
\text { LASER }\end{array}$ & & & & & & \\
\hline CAMPANHA & & & & & & \\
\hline BRUTO & & & & & & \\
\hline DINHEIRO & & & & & & \\
\hline $\begin{array}{l}\text { CARTÃO DE } \\
\text { CRÉDITO }\end{array}$ & & & & & & \\
\hline $\begin{array}{l}\text { CARTÃO DE } \\
\text { DÉBITO }\end{array}$ & & & & & & \\
\hline DEPÓSITOS & & & & & & \\
\hline CANCELAMENTC & & & & & & \\
\hline LÍQUIDO & & & & & & \\
\hline COFRE & & & & & & \\
\hline OUANTIDADE & TOTAL & META & MÉDIA & $\begin{array}{c}\text { META } \\
\text { POR } \\
\text { DIA }\end{array}$ & ATINGIMENTO & $\begin{array}{c}\text { COMO } \\
\text { ESTAMOS? }\end{array}$ \\
\hline VENDAS & & & & & & \\
\hline MANUNTENÇÕES & & & & & & \\
\hline RASPAGENS & & & & & & \\
\hline INST.INFERIOR & & & & & & \\
\hline CLAREAMENTO & & & & & & \\
\hline IRAX & & & & & & \\
\hline KITS & & & & & & \\
\hline $\begin{array}{l}\text { PACIENTES } \\
\text { ATIVOS }\end{array}$ & & & & & & \\
\hline $\begin{array}{l}\text { PACIENTES } \\
\text { INATIVOS }\end{array}$ & & & & & & \\
\hline ATENDIMENTOS & & & & & & \\
\hline AVALIAÇÕES & & & & & & \\
\hline URGENCIAS & & & & & & \\
\hline
\end{tabular}

Fonte: Elaboração Própria (2019)

A figura 6 representa a planilha online de 2019.1 que além da quantidade tem os valores separados por fonte de receita, também foi adicionada a meta mensal com o atingimento, o quanto seria necessário fazer por dia útil, a média dos dias realizados e como estavam. $\mathrm{O}$ 
controle auxiliou na clareza de como a clínica estava após o dia realizado e na dinâmica de reuniões semanais e mensais, tendo maior facilidade para identificação de pontos de melhoria e aplicação de planos de ação criando agilidade nos processos dentro da companhia.

Em outubro de 2019 foi realizado o segundo ciclo de ajuste dos objetivos e indicadores. Utilizando os dados monetários e quantitativos da média dos meses do primeiro, segundo e terceiro trimestres do ano como apresentados abaixo:

Tabela 4 - Realizado Niterói

\begin{tabular}{|c|c|c|c|c|c|c|}
\hline NITEROI & $1^{\circ}$ TRI 2019 & $\%$ & $2^{\circ}$ TRI 2019 & $\%$ & $\begin{array}{c}3^{\circ} \text { TRI } \\
2019\end{array}$ & $\%$ \\
\hline RECEITA BRUTA & $\mathrm{R} \$ 122.929,66$ & & $\mathrm{R} \$ 142.033,46$ & & $\mathrm{R} \$ 155.108,1$ & \\
\hline VENDAS/MRR & $\mathrm{R} \$ 23.770,03$ & & $\mathrm{R} \$ 21.208,83$ & & $\mathrm{R} \$ 19.449,8$ & \\
\hline $1^{\circ}$ RETORNO & $\mathrm{R} \$ 14.630,42$ & & $\mathrm{R} \$ 16.776,02$ & & $\mathrm{R} \$ 14.368,7$ & \\
\hline DEMAIS RETORNOS & $\mathrm{R} \$ 76.629,18$ & & $\mathrm{R} \$ 92.247,83$ & & $\mathrm{R} \$ 107.682,2$ & \\
\hline RASPAGENS & $\mathrm{R} \$ 7.338,30$ & & $\mathrm{R} \$ 9.041,63$ & & $\mathrm{R} \$ 8.790,0$ & \\
\hline $\mathrm{N}^{\circ} \mathrm{DE}$ VENDAS & 239 & & 218 & & 200 & \\
\hline $\mathrm{N}^{\circ}$ DE $1^{\circ}$ RETORNO & 164 & $66 \%$ & 185 & $80 \%$ & 156 & $85 \%$ \\
\hline $\mathrm{N}^{\circ}$ DE DEMAIS RETORNOS & 846 & $90 \%$ & 1.024 & $89 \%$ & 1.182 & $89 \%$ \\
\hline RASPAGENS & 135 & $56 \%$ & 170 & $78 \%$ & 153 & $77 \%$ \\
\hline
\end{tabular}

Fonte: Elaboração Própria (2019)

$\mathrm{Na}$ tabela 4, a receita bruta cresceu nos três trimestres comprovando o modelo de retenção e maturidade da clínica que está no segundo ano. As vendas tanto em valor quanto quantidade apresentam queda nos três trimestres consecutivos, sendo necessária atenção neste fator no próximo ciclo. Na parte de primeiro retorno a clínica apresenta crescimento gradual, atingindo as metas do OKR no terceiro trimestre. Nos demais retornos a clínica atinge a meta de $90 \%$ no primeiro trimestre e alcança nos segundos e terceiros trimestres o percentual de $89 \%$ de retenção, indicando o progresso da receita bruta pela alta retenção do primeiro retorno e demais retornos comprovando o modelo de recorrência. $\mathrm{O}$ indicador de raspagem tem grande desenvolvimento, passando de $56 \%$ da venda para $78 \%$, discriminando o efeito da clareza e dos planos de ação dentro da clínica. 


\begin{tabular}{l|c|c|c|c}
\hline \multicolumn{1}{c|}{ NITEROI } & $\begin{array}{c}\text { META } \\
\mathbf{2 0 1 9 . 1}\end{array}$ & $\begin{array}{c}\mathbf{1}^{\circ} \text { TRI } \\
\mathbf{2 0 1 9}\end{array}$ & $\begin{array}{c}\mathbf{2}^{\circ} \text { TRI } \\
\mathbf{2 0 1 9}\end{array}$ & $\begin{array}{c}3^{\circ} \text { TRI } \\
\mathbf{2 0 1 9}\end{array}$ \\
\hline $\mathrm{N}^{\circ}$ DE VENDAS & 250 & $96 \%$ & $87 \%$ & $80 \%$ \\
\hline $\mathrm{N}^{\circ}$ DE 1 ${ }^{\circ}$ RETORNO & $85 \%$ & $78 \%$ & $95 \%$ & $100 \%$ \\
\hline $\mathrm{N}^{\circ}$ DE DEMAIS & $90 \%$ & $100 \%$ & $99 \%$ & $99 \%$ \\
RETORNOS & & & & \\
\hline RASPAGENS & $75 \%$ & $75 \%$ & $104 \%$ & $102 \%$ \\
\hline
\end{tabular}

Fonte: Elaboração Própria (2019)

Na tabela 5 é exposto o atingimento percentual dos trimestres a partir da meta de 2019.1. A evolução no primeiro, segundo e terceiro trimestres comprova que metas bem definidas e claras auxilia o objetivo a ser alcançado. Na tabela 6 , ocorre a comparação do início do estudo no último trimestre de 2018 com o terceiro trimestre de 2019.

Tabela 6 - $4^{\circ}$ TRI 2018 X $3^{\circ}$ TRI 2019 NITERÓI

\begin{tabular}{|c|c|c|c|c|c|}
\hline NITEROI & $\begin{array}{c}4^{\circ} \text { TRI } \\
2018\end{array}$ & $\%$ & $\begin{array}{c}3^{\circ} \text { TRI } \\
2019\end{array}$ & $\%$ & $\begin{array}{c}4^{\circ} \mathrm{T} 2018 \times 3^{\circ} \mathrm{T} \\
2019\end{array}$ \\
\hline $\mathrm{N}^{\circ} \mathrm{DE}$ VENDAS & 224 & & 212 & & $-5 \%$ \\
\hline $\mathrm{N}^{\circ} \mathrm{DE} 1^{\circ} \mathrm{RETORNO}$ & 165 & $77 \%$ & 160 & $81 \%$ & $4 \%$ \\
\hline $\begin{array}{l}\mathrm{N}^{\circ} \text { DE DEMAIS } \\
\text { RETORNOS }\end{array}$ & 597 & $86 \%$ & 890 & $87 \%$ & $1 \%$ \\
\hline RASPAGENS & 125 & $55 \%$ & 139 & $66 \%$ & $11 \%$ \\
\hline
\end{tabular}

Fonte: Elaboração Própria (2019)

Conforme apresentado na tabela 6, o terceiro trimestre de 2019 só decai em número de vendas, porém nos indicadores de número de primeiro retorno, demais retornos e raspagens têm efeitos benéficos do estudo dentro da clínica de Niterói.

Com isso, para os próximos objetivos e metas de médio prazo, se deve levar em consideração uma padronização da venda com processos bem definidos, checklists e treinamentos para a área comercial, visto que existe uma discrepância comercial grande entre as clínicas. Além de um controle maior da retenção, identificando os microproblemas no processo entre a venda e o primeiro retorno para conseguir explicar e solucionar a dificuldade de reter o paciente na venda e controlar a nível de usuário os indicadores do estudo potencializando o desempenho por colaborador. 
Para plano de longo prazo, automatizar os controles e internalizar no sistema os indicadores, criando maior agilidade e clareza para cada ponta e usuário do processo. Criar também todos os manuais de usuários e estender o estudo para todas as áreas da clínica e para um controle de despesa com metas de atingimento.

\section{Conclusão}

$\mathrm{O}$ artigo tem como finalidade analisar a implantação da metodologia OKR e seus desafios dentro de uma pequena empresa do ramo odontológico, visto a importância do setor de saúde na economia nacional e o momento de retomada que ele se encontra.

O trabalho foi desenvolvido através de um estudo de caso onde acompanhou-se a sua implementação da metodologia e seus respectivos impactos dentro da clínica de Niterói, o qual geraram um aumento nos indicadores de primeiro retorno, manutenções e raspagens. Além do aumento, foi identificado uma melhora na clareza e comunicação sobre metas e objetivos a serem alcançados criando maior agilidade e foco nos processos dentro da clínica. Portanto, a metodologia OKR pode ser facilmente aplicada em micro e pequenas empresas como apresentado, provocando melhoras significativas na clareza, agilidade, comunicação, definição de metas e objetivos, além de potencializar o resultado. Seria assim, um método de muita valia para os empreendedores, para a sobrevivência e desenvolvimento de novos negócios.

\section{REFERÊNCIAS}

AGUiAR, S. Integração das Ferramentas da Qualidade ao PDCA e ao Programa Seis Sigma. Belo Horizonte: Editora de Desenvolvimento Gerencial, 2002.

BETTERWORKS. Getting started with objectives \& key results (OKRs): best practices for implementing OKRs in your business. California: [s.n.], 2015.

CASTRO, F. The Beginners Guide to OKR. The Beginners Guide to OKR, 2017. Disponivel em: 〈https://felipecastro.com/resource/The-Beginners-Guide-to-OKR.pdf>.

DOERR, J. Avalie o que Importa. [S.1.]: Alta Books, 2019.

ESTATÍSTICAS ECONÔMICAS. Demografia das Empresas e Empreendedorismo 2016: taxa de entrada fica em $14,5 \%$ e é a menor desde 2008. IBGE, 3 out. 2018. Disponivel em: $<$ https://agenciadenoticias.ibge.gov.br/agencia-sala-de-imprensa/2013-agencia-de-noticias/releases/22710demografia-das-empresas-e-empreendedorismo-2016-taxa-de-entrada-fica-em-14-5-e-e-a-menor-desde-2008>. IBGE. Brasil enfrenta pior crise já registrada poucos anos após um boom econômico. G1, 07 mar. 2017. Disponivel em: <https://g1.globo.com/economia/noticia/brasil-enfrenta-pior-crise-ja-registrada-poucos-anosapos-um-boom-economico.ghtml>. 
LAMORTE B. Objectives and Key Results: tips from an OKRs coach. [S.1.]: [s.n.], 2015.

NIVEN, P. R., LAMORTE, B. Objectives and Key Results: Driving Focus, Alignment, and Engagement with OKRs. [S.1.]: Wiley Corporate F\&A, 2016.

SEBRAE. Boletins de Estudos \& Pesquisas - Ano 2017. Sebrae, 2017. Disponivel em: <http://www.sebrae.com.br/Sebrae/Portal\%20Sebrae/Anexos/7836>.

GATES, S. Aligning Strategic Performance Measures and Results. New York: The Conference Board, 1999.

SEBRAE. Sobrevivencia das empresas no Brasil. Sebrae, out. 2016. Disponivel em: <http://www.sebrae.com.br/Sebrae/Portal\%20Sebrae/Anexos/sobrevivencia-das-empresas-no-brasil102016.pdf>.

TERESA M M MACIEL, DANIEL ARCOVERDE. Gestão por Objetivos e Resultados com OKR. Gestão por Objetivos e Resultados com OKR. Disponivel em: <http://www.xiwticifes.ufba.br/modulos/submissao/Upload353/86125.pdf>.

WODTKE, C. Introduction to OKRs. California: O’Reilly Media, 2016. 